04

\title{
Пороговый характер раскручивания объемного пылевого кластера в магнитном поле
}

\author{
() В.Ю. Карасев, Е.С. Дзлиева, С.И. Павлов, Л.А. Новиков, А.И. Эйхвальд, И.Ч. Машек \\ Санкт-Петербургский государственный университет, \\ 199034 Санкт-Петербург, Россия \\ e-mail: plasmadust@yandex.ru, v.karasev@spbu.ru
}

Поступило в Редакцию 11 июля 2019 г.

В окончательной редакции 11 июля 2019 г.

Принято к публикации 13 июля 2019 г.

\begin{abstract}
Представлено исследование воздействия магнитного поля на поведение объемного пылевого кластера, образованного в страте тлеющего разряда. Изучен кластер, состоящий из семи цепочек частиц и представляющий собой в горизонтальном сечении гексагональную ячейку. Обнаружено, что возникновение вращения пылевого кластера происходит в ненулевом магнитном поле в отличие от пылевой структуры, содержащей большее число частиц в горизонтальном сечении. Кроме того, обнаружено, что на пороговый характер возникновения вращения в кластере влияет разрядный ток и давление плазмаформирующего газа. В качестве интерпретации выдвинуто предположение об увеличении ионизации внутри пылевой структуры. Радиальный поток ионов, идущий в продольном магнитном поле из пылевой структуры к стенке разрядной трубки, дополнительно раскручивает структуру с отрицательной проекцией угловой скорости на вектор магнитной индукции.
\end{abstract}

Ключевые слова: пылевая плазма, магнитное поле, тлеющий разряд, кластеры.

DOI: $10.21883 /$ JTF.2020.02.48809.269-19

\section{Введение}

Пылевая плазма обладает множеством уникальных значений физических величин (молярная масса, заряд, энергия взаимодействия между частицами и др.), что обеспечивает ее экстремальные электрические, магнитные, термодинамические и механические свойства [1-4]. Одной из экспериментально обнаруженных особенностей ее механических свойств является проявление пороговых эффектов. Среди них известны порог возникновения течения в пылевом образовании при лазерном сдвиговом воздействии $[5,6]$, появление собственного вращения у уединенных пылевых частиц (возникновение у них магнитных моментов) [7,8], а также масштабные эффекты (зависимость от числа частиц, размера структуры) появления вращательного движения пылевой структуры в магнитном поле $[9,10]$.

Последний эффект интересно исследовать в пылевой ловушке в тлеющем разряде, где интенсивность плазменных потоков выше чем в ВЧ разряде, и можно использовать объемные пылевые структуры. Магнитное поле может быть применено для визуализации действия потока ионов в азимутальном направлении. Угловая скорость будет служить количественной мерой силового воздействия такого потока и изменения параметров плазмы.

Зависимость возникновения вращения от числа пылевых частиц убедительно показана в монослойной пылевой структуре [10]. В объемных пылевых структурах каждая новая инжектированная частица может изменить объемную пылевую ячейку, меняя не только ее геомет- рию перпендикулярно магнитному полю, но и в продольном направлении, дополнительно изменяя ее объем. Поэтому в настоящей работе мы изучаем зависимость возникновения вращения в магнитном поле в малой по объему пылевой структуре, состоящей лишь из одной гексагональной ячейки в горизонтальном сечении, от таких параметров газового разряда, как ток и давление газа. Для более тонкого воздействия (минимизации потока плазмы) применены мелкие частицы, но с достаточно большой плотностью для обеспечения их устойчивой левитации в кластере.

\section{Эксперимент и результаты}

Для создания требуемого плазменно-пылевого образования, схематически изображенного на рис. 1, в качестве пылевой ловушки использовалась стоячая страта в разряде неона при давлении $p=(0.5-1.0) \cdot 10^{2} \mathrm{~Pa}$ и токах разряда $i=1.6-2.2 \mathrm{~mA}$. Для формирования пылевой компоненты использовались частицы ниобата лития плотностью $4.6 \mathrm{~g} / \mathrm{cm}^{3}$, диаметром $2 \mu \mathrm{m}$ и формы, близкой к сферической.

В общих чертах экспериментальная установка была описана ранее в $[8,11]$. Для исследования использовалась разрядная трубка радиусом $1 \mathrm{~cm}$, снабженная никелевыми электродами, конструктивно вынесенными из области магнитного поля. Для наложения магнитного поля трубка располагалась между парой магнитных катушек. Для визуализации производилась боковая подсветка одного горизонтального сечения структуры лазерным 


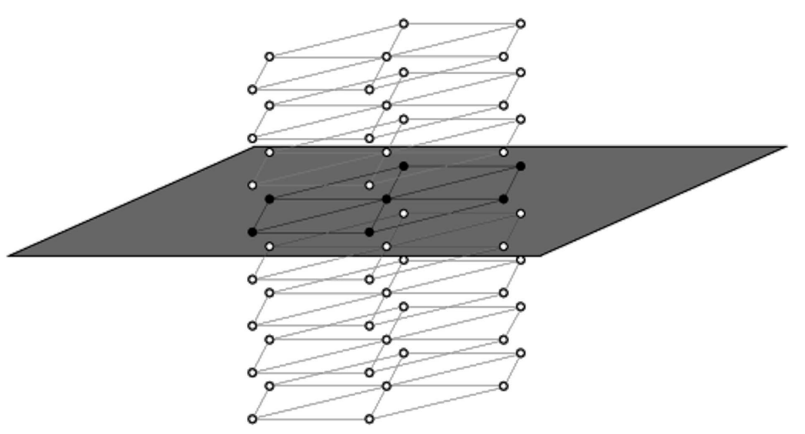

Рис. 1. Схема объемного кластера, состоящего из 7 вертикально ориентированных пылевых цепочек (7 частиц в горизонтальном сечении), сконструированного в эксперименте.

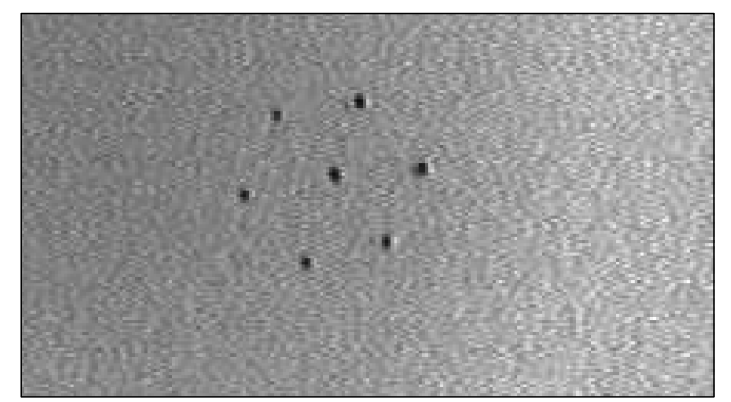

Рис. 2. Инвертированная фотография горизонтального сечения пылевого кластера, созданного в пылевой ловушке в страте. Условия: газ неон, $p=10^{2} \mathrm{~Pa}, i=1 \mathrm{~mA}$, частицы ниобата лития диаметром $2 \mu \mathrm{m}$. Размер рисунка по горизонтали $3.8 \mathrm{~mm}$.

ножом. Видеозапись рассеянного пылевыми частицами излучения производилась через торцевое окно разрядной трубки.

Для создания пылевой структуры из малого количества пылевых цепочек использовался метод инжектирования частиц, описанный в [7,12], контейнер с пылевыми частицами подвешивался в верхней части разрядной трубки. Пример изображения получаемого горизонтального сечения исследованной пылевой структуры без магнитного поля показан на рис. 2. При медленном наложении магнитного поля пылевое образование приходит во вращение.

Порог возникновения вращения фиксировался по зависимости угловой скорости от магнитной индукции, примеры которой для нескольких условий представлены на рис. 3. Сопоставление рис. 3, $a$ и $b$ отражает зависимость магнитного поля, при котором возникает вращение, от числа частиц в горизонтальном сечении пылевой структуры. В исследуемом кластере вращение развивается после $50 \mathrm{G}$, в то время как в протяженной структуре оно начинается от нулевого значения поля. Сопоставление рис. 3, $a$ и $c$ демонстрирует зависимость порогового поля от тока разряда. В выбранных условиях увеличение тока до $2.2 \mathrm{~mA}$ приводит к отсутствию порога возникновения. Сопоставление рис. $3, a$ и $d$ указывает на зависимость порога возникновения вращения от давления газа. По всем проведенным измерениям данная зависимость выражена более слабо, чем зависимость от числа частиц и тока разряда.

\section{Обсуждение}

Вращение пылевой структуры в магнитном поле используемого диапазона (в наших условиях до $100 \mathrm{G}$ [11]) вызывается силой ионного увлечения. Радиальный поток ионов, идущий к стенке разрядной трубки в продольном магнитном поле, раскручивает пылевую структуру с отрицательной проекцией угловой скорости на вектор магнитной индукции. Баланс силы ионного увлечения и силы сопротивления Эпштейна [1-4] для отдельной пылевой частицы дает выражение угловой скорости вращения

$$
\omega=\frac{n_{i} m_{i} \vartheta_{T i}\left(\omega_{i} \tau_{i}\right) U_{i r} \vartheta_{T n}\left\{1+\frac{z \tau}{2}+\frac{z^{2} \tau^{2}}{4} \Pi\right\}}{p r_{d}} .
$$

Здесь $a$ - радиус частицы, $r_{d}$ - радиус ее вращения, $\tau=\frac{T_{e}}{T_{i}}, z=\frac{z_{d} e^{2}}{a T_{e}}-$ безразмерный заряд, $Z_{d}-$ зарядовое число, П - модифицированный кулоновский логарифм [1-4], $\omega_{i} \tau_{i}$ - произведение циклотронной частоты иона на время между столкновениями, остальные обозначения стандартные. Формула (1) дает соответствующее эксперименту значение скорости вращения, но не показывает порогового характера его развития. В стационарном потоке ионов вращение будет инициироваться постепенно от нулевого магнитного поля. При подобранных в эксперименте условиях (так же как с плоскими малыми кластерами в ВЧ разряде [10]) регистрируется пороговый характер возникновения вращения. Существование пороговых механических явлений в пылевой плазме известно по работам $[5,6,8,10]$. Например, в последней возникновение собственного вращения частиц не связано с полным током разряда, а вызывается потоком плазмы, идущим непосредственно на пылевую частицу в процессе поддержания стационарного заряда на ее поверхности.

Объясняя обнаруженное пороговое раскручивание пылевого кластера (рис. 3,a,c), мы связываем его с дополнительным потоком ионов. В присутствии пылевой подсистемы в плазме появляется вторая составляющая радиального потока. Первая представляет собой обычный амбиполярный поток [13], вторая - поток, возникающий из-за дополнительной ионизации внутри пылевой структуры. На пылевых частицах происходит рекомбинация, и в области их левитации возрастает электрическое поле и электронная температура, как было установлено в [14]. Как следствие, внутри пылевой структуры возрастает частота ионизации, компенсирующая рекомбинацию. Потери плазменных частиц на пылевой частице оцениваются из выражения для зондового 

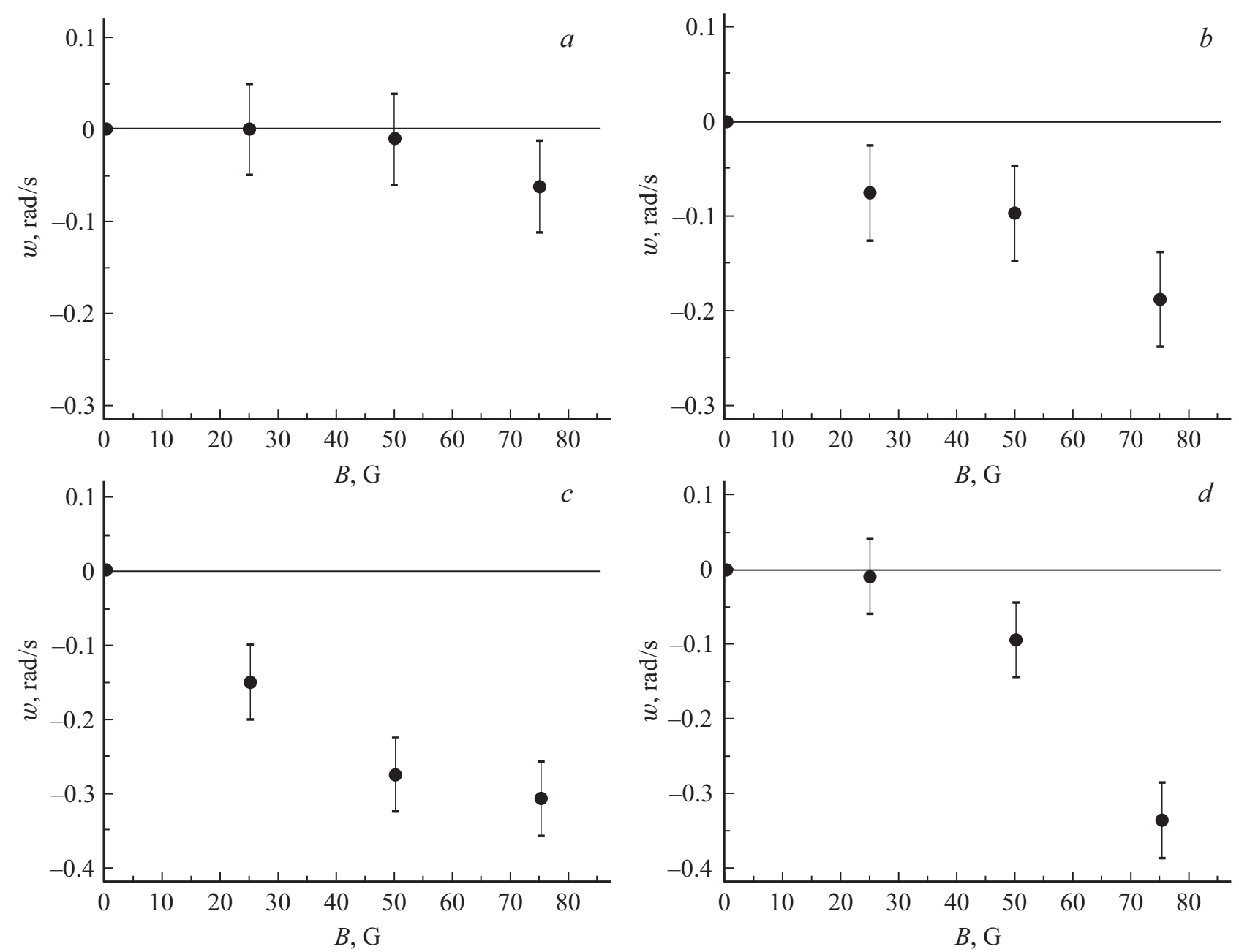

Рис. 3. Зависимость угловой скорости вращения горизонтального сечения пылевого образования от магнитного поля: $a-$ пылевой кластер (7 частиц в сечении), $p=0.5 \cdot 10^{2} \mathrm{~Pa}, i=1.6 \mathrm{~mA} ; b-$ среднее по вертикали горизонтальное сечение пылевой структуры (30 частиц в сечении), $p=0.5 \cdot 10^{2} \mathrm{~Pa}, i=1.6 \mathrm{~mA} ; c$ - пылевой кластер, $p=p=0.5 \cdot 10^{2} \mathrm{~Pa}, i=2.2 \mathrm{~mA} ; d-$ пылевой кластер, $p=p=0.8 \cdot 10^{2} \mathrm{~Pa}, i=2.2 \mathrm{~mA}$.

тока ионов при плавающем потенциале [15]:

$$
I_{i}=e=\sqrt{8 \pi} a^{2} n_{i} v_{T i}\left(1+\frac{e U}{k T_{i}}\right),
$$

где $e-$ заряд электрона, $U-$ потенциал пылевой частицы. Для условий проводимого эксперимента число рекомбинирующих на поверхности одной пылевой частицы ионов и электронов за одну секунду порядка $10^{8}$. Внутри объемной пылевой структуры оно может быть больше на несколько порядков. Добавление в структуру каждой пылевой частицы будет дискретно увеличивать рассматриваемый поток, генерируя появление вращения в магнитном поле.

В созданном пылевом кластере плотность пылевой компоненты порядка $n_{d}=10^{5} \mathrm{~cm}^{-3}$ и доминирующим каналом потерь плазмы является рекомбинация на пылевой структуре. Из условия квазинейтральности при наличии пылевых частиц $n_{i}=n_{e}+Z_{d} n_{d}$, можно ожидать появления радиального потока ионов через границу пылевой структуры, пропорционального $\Delta n=n_{i}-n_{i 0}$, где $n_{i 0}$ - плотность ионов вне пылевого кластера. Сoгласно выполненным оценкам, данный поток вызывает вращение пылевой структуры по формуле (1) с угловой скоростью до $1 \mathrm{rad} / \mathrm{s}$. Таким образом, предложенная интерпретация объясняет пороговый характер возникновения вращения пылевой структуры при увеличении числа пылевых частиц.

При увеличении тока разряда вращение развивается как согласно (1), так и согласно (2) из-за увеличения концентрации ионов $n_{i 0}$, и пороговый характер будет проявляться. Зависимость же порога возникновения вращения от давления проявляется слабо (рис. 1,d). Можно предположить, что она может быть связана с малым увеличением электронной температуры по мере уменьшения давления. Выполненная оценка на основе универсальной кривой для вычисления $T_{e}[13]$ для наших условий дает возможное увеличение электронной темпе- 
ратуры порядка 5\%, весьма малое, даже по сравнению с погрешностью ее определения.

\section{Заключение}

Впервые исследовано пороговое возникновение вращательного движения объемной пылевой структуры в магнитном поле. Сопоставлено возникновение вращения в симметрично организованном (гексагональном) пылевом кластере и большой структуре. Установлено наличие порога возникновения вращения от числа частиц, тока разряда и давления газа. Предложена интерпретация, показывающая, что причиной возникновения вращения в данных условиях является радиальная компонента потока ионов в магнитном поле, вызванная увеличением электронной температуры внутри пылевой структуры.

\section{Финансирование работы}

Работа поддержана Российский Научный Фонд (РНФ), грант № 18-72-10019, часть расходных материалов и оборудования были получены по программе модернизации материальной и технической базы научных исследований Санкт-Петербургского государственного университета (СПбГУ).

\section{Конфликт интересов}

Авторы заявляют, что у них нет конфликта интересов.

\section{Список литературы}

[1] Fortov V.E., Mofill G.E. Complex and Dusty Plasmas: from Laboratory to Space. NY:: Taylor \& Francis Group, 2010. $440 \mathrm{p}$.

[2] Vladimirov S.V., Ostrikov K., Samarian A.A. Physics and Applications of Complex Plasmas. London: Imperial College Press, 2005. $439 \mathrm{p}$.

[3] Tsytovich V.N., Morfill G.E., Vladimirov S.V., Thomas H.M. Elementary Physics of Complex Plasmas. NY.: Springer, 2008. $370 \mathrm{p}$.

[4] Bonitz M., Horing N., Ludwig P. Introduction to Complex Plasma. Springer-Verlag: Berlin Heidelberg, 2010. 443 p.

[5] Ворона Н.А., Гавриков А.В., Иванов А.С., Петров О.Ф., Фортов В.Е., Шахова И.А. // ЖЭТФ. 2007. Т. 132. С. 941.

[6] Gavrikov A.V., Goranskaya D.N., Ivanov A.S., Petrov O.F., Timirkhanov R.A., Vorona N.A., Fortov V.E. // J. Plasma Phys. 2010. Vol. 76. N 3-4. P. 579.

[7] Karasev V.Yu., Dzlieva E.S., Eikhval'd A.I., Ermolenko M.A., Golubev M.S., Ivanov A.Yu. // Phys. Rev. E. 2009. Vol. 79. P. 026406.

[8] Карасев В.Ю., Ермоленко М.А., Дзлиева Е.С., Павлов С.И., Новиков Л.А., Машек И.Ч. // ЖТФ. 2016. Т. 86. Вып. 4. С. 139.

[9] Sato N. // AIP Conf. Proc. 2002. Vol. 649. P. 66.

[10] Cheung F., Samarian Al., James B. // New J. Phys. 2003. Vol. 5. P. 75.

[11] Karasev V.Yu., Dzlieva E.S., Ivanov A.Yu., Eikhval'd A.I. // Phys. Rev. E. 2006. Vol. 74. P. 066403.
[12] Golubovskii Y., Karasev V., Kartasheva A. // Plasma Sources Sci. Technol. 2017. Vol. 26. P. 115003.

[13] Райзер Ю.П. Физика газового разряда. М.: Наука, 1992. $536 \mathrm{c}$.

[14] Липаев А.М., Молотков В.И., Нефедов А.П., Петров О.Ф., Торчинский В.М., Фортов В.Е., Храпак А.Г., Храпак С.А. // ЖЭТФ. 1997. Т. 112. С. 2030.

[15] Chen F.F. Electric Probes. Plasma Diagnostic Techniques. NY: Academic Press, 1965. 200 p. 